\title{
High-Energy Cutoff in the Spectrum of Strong-Field Nonsequential Double Ionization
}

\author{
J. S. Parker, ${ }^{1}$ B. J. S. Doherty, ${ }^{1}$ K. T. Taylor,${ }^{1}$ K. D. Schultz, ${ }^{2}$ C. I. Blaga, ${ }^{2}$ and L. F. DiMauro ${ }^{2}$ \\ ${ }^{1}$ DAMTP, Queen's University Belfast, Belfast, BT7 1NN, United Kingdom \\ ${ }^{2}$ Department of Physics, The Ohio State University, Columbus, Ohio 43210, USA
}

(Received 13 December 2005; published 6 April 2006)

\begin{abstract}
Electron energy distributions of singly and doubly ionized helium in an intense $390 \mathrm{~nm}$ laser field have been measured at two intensities $\left(0.8 \mathrm{PW} / \mathrm{cm}^{2}\right.$ and $1.1 \mathrm{PW} / \mathrm{cm}^{2}$, where $\left.\mathrm{PW} \equiv 10^{15} \mathrm{~W} / \mathrm{cm}^{2}\right)$. Numerical solutions of the full-dimensional time-dependent helium Schrödinger equation show excellent agreement with the experimental measurements. The high-energy portion of the two-electron energy distributions reveals an unexpected $5 U_{p}$ cutoff for the double ionization (DI) process and leads to a proposed model for DI below the quasiclassical threshold.
\end{abstract}

DOI: 10.1103/PhysRevLett.96.133001

PACS numbers: $32.80 . \mathrm{Fb}, 31.90 .+\mathrm{s}, 32.80 . \mathrm{Rm}$

In an intense low-frequency laser field, the mechanism for double ionization of helium varies with intensity. At the highest intensities, the process can be accurately described by a sequence of independent, single-electron ionization steps [dubbed sequential double ionization (SDI) [1]], in which the atom is singly ionized first, followed by photoionization of the residual $\mathrm{He}^{+}$ion. This process produces uncorrelated pairs of electrons. The rate at which this process occurs is limited by the slower of the two steps, typically the ionization of the residual $\mathrm{He}^{+}$ion. In helium, at visible and near-infrared (IR) wavelengths, SDI becomes negligible at intensities $\leq 4 \mathrm{PW} / \mathrm{cm}^{2}$. Below this intensity, $\mathrm{He}^{+}$is produced predominantly via nonsequential double ionization (NSDI), a process in which correlated electron pairs are ejected near simultaneously ( $\leq 1$ optical cycle). In the near IR, NSDI is well described by a quasiclassical rescattering model [2,3], in which a single electron is ejected at or near the peak of the electric field, but returns to the atom as the field changes direction, and does so with sufficient energy to free the remaining electron of $\mathrm{He}^{+}$in an inelastic $(e, 2 e)$ collision. The maximium energy the rescattered electron can bring to the $\mathrm{He}^{+}$ion is $3.2 U_{p}$, where $U_{p} \propto I \lambda^{2}$ is the ponderomotive energy. The threshold intensity at which the rescattered electron has sufficient energy to directly ionize the $\mathrm{He}^{+}(1 s)$ is the intensity at which $3.2 U_{p}$ equals the ionization potential of $\mathrm{He}^{+}: 2$ au. We label this intensity $I_{t 1}$. Below $I_{t 1}$ the rescattered electron has insufficient energy to directly ionize $\mathrm{He}^{+}(1 s)$, but if the intensity is above $I_{t 2}=0.75 I_{t 1}$, it does have sufficient energy to collisionally excite the first excited state of $\mathrm{He}^{+}$, which can then ionize rapidly, absorbing energy from the laser. Although this is a plausible mechanism for NSDI in the region $I_{t 2}<I<I_{t 1}$ it has not been established if it is the only mechanism, or whether it is the dominant NSDI process. Below the $I_{t 2}$ threshold the physical origins of NSDI have remained puzzling [4]. Recently, experimental progress [5] has been reported in this intensity limit, but for higher- $Z$ inert gases excited by $800 \mathrm{~nm}$ light.

In this Letter we report on an experimental and theoretical double ionization study of helium atoms in the intensity range $I<I_{t 2}$ and $I_{t 2}<I<I_{t 1}$ using intense $390 \mathrm{~nm}$ light. We propose a possible mechanism for NSDI in this regime utilizing the advantages enabled by the shorter, near-UV wavelength excitation. First, the thresholds $\left(I_{t 1}, I_{t 2}\right)$ are wavelength dependent due to the $\lambda^{2}$ scaling of the ponderomotive energy $U_{p}$. For example, at $390 \mathrm{~nm} I_{t 2}=$ $0.9 \mathrm{PW} / \mathrm{cm}^{2}$, while at $780 \mathrm{~nm}, I_{t 2}=0.225 \mathrm{PW} / \mathrm{cm}^{2}$. At $I=I_{t 2}$ the ratio of double to single ionization is an order of magnitude higher at $390 \mathrm{~nm}$ than it is at $780 \mathrm{~nm}$, and total ionization yield many orders of magnitude higher. This is crucial since the double ionization measurement relies upon electron-ion coincidence for discriminating against the more abundant single-electron events. Coincidence methods are inherently a low duty cycle measurement and thus the experiments benefit from a single shot count rate of near unity. In addition, $780 \mathrm{~nm}$ light is equally unsuitable for theoretical calculations, since the computational overhead scales as $\lambda^{3}$. The greater computational efficiency at $390 \mathrm{~nm}$ enables high accuracy numerical solutions of the full-dimensional 2-electron Schrödinger equation for the helium atom in a laser field, and a detailed analysis of NSDI in the range $0.4-1.6 \mathrm{PW} / \mathrm{cm}^{2}$. Consequently, $390 \mathrm{~nm}$ excitation of helium provides an excellent meeting ground for theory and experiment in the exploration of multielectron intense-field processes.

The coincidence spectrometer has been discussed in depth elsewhere [6,7]. Briefly, the $390 \mathrm{~nm}$ light is produced by frequency doubling in a $0.5 \mathrm{~mm}$ thick $\mathrm{BBO}\left(\beta-\mathrm{BaB}_{2} \mathrm{O}_{4}\right)$ crystal the output of a $\sim 120 \mathrm{fs}, 780 \mathrm{~nm}$ regenerative amplified titanium sapphire laser. The light is focused into an ultrahigh vacuum chamber at repetition rates of $1-2 \mathrm{kHz}$. The pulse energy is adjusted to obtain the desired peak intensity and the gas pressure is varied to obtain an appropriate count rate. The spectrometer is a pulsed-plate dual-sided time-of-flight (TOF) design which measures the electron energy and ion $m / q$ distribution for each laser shot. The ${ }^{3} \mathrm{He}$ isotope is used to remove the near $\mathrm{m} / \mathrm{q}$ degeneracy of the ${ }^{4} \mathrm{He}^{2+}$ and $\mathrm{H}_{2}^{+}$ions. In coincidence mode, the instrument allows the detected electron(s) to be correlated with a particular ion. The resolution of the 
electron energy analyzer and mass spectrometer is 5\% and $1 / 300$, respectively. It is important to note that we cannot distinguish electrons, and therefore the measured double ionization distribution corresponds to the total electron yield.

The ratio of true-to-false (T:F) coincidences can be calculated if the efficiencies and the ionization count rates are known [8]. In this experiment the efficiencies of ion and electron detection are $30 \%$ and $1 \%$, respectively, and the count rate is $\leq 0.5$ ion per shot. In Fig. 1(a) $10^{8}$ laser shots resulted in $3.6 \times 10^{6}$ single ionization events and 1262 double ionization events. At this intensity, the T:F ratio is 13.0 and 1.8, respectively. In Fig. 1(b) $10^{8}$ laser shots are taken, yielding $2.9 \times 10^{6}$ single ionization events and 2129 double ionization events. The T:F ratio is 11.3 and 1.8, respectively. It should be noted that for high momenta electrons, where the production of "false" electrons is very low, the $T: F$ ratio is much greater. The experimental data presented in Fig. 1 are not corrected for false coincidences, since this only results in small changes to the low momenta part of the distribution.

Details of the numerical methods are described elsewhere [9]. The full-scale integration were performed using a variety of pulse parameters that included 7 or 8 field period pulses, cosine and sine phases, and ramp ons of 1.5, 2 , and 3 field periods. Variation of these and other parameters produced insignificant changes in the numerical result. At the end of the pulse, the doubly ionizing wave packets are extracted from the wave function using masks, and $\psi$ is transformed into momentum space. The angular variables of $\psi^{*} \psi$ are integrated away to leave the joint-probability distribution of the electrons as a function of the radial momenta $p_{1}, p_{2}$, so that total kinetic energy in atomic units is $E=p_{1}^{2} / 2+p_{2}^{2} / 2$.

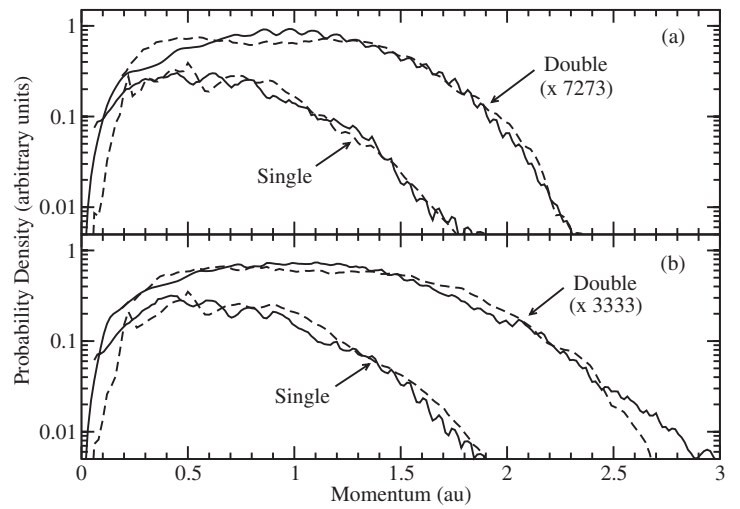

FIG. 1. Electron momentum distributions at $390 \mathrm{~nm}$ for singly and doubly ionized helium at (a) $0.8 \mathrm{PW} / \mathrm{cm}^{2}$ and (b) $1.1 \mathrm{PW} / \mathrm{cm}^{2}<I_{t 1}=1.2 \mathrm{PW} / \mathrm{cm}^{2}$. Numerical integration and experiment are shown as solid and dashed lines, respectively. In the case of double ionization, plotted is the momentum of just one of the ionized electrons, determined independently of the other.
In the case of single ionization it is possible to model the complex geometry of the experiment by using a singleactive electron (SAE) model [10]. The SAE code is tuned to give quantitative agreement with the full-scale helium integration, but is several orders of magnitude more efficient, enabling more complete modeling of the experimental $120 \mathrm{fsec}$ pulse. The integrations are performed at 64 different intensities in the range $0.4-1.2 \mathrm{PW} / \mathrm{cm}^{2}$, and the results are averaged over the spatial intensity distribution of a Gaussian focus.

Figure 1 compares theoretical and experimental results on a $\log$ scale to emphasize the high-end exponential decays of the distribution at two different laser intensities. The momentum measurements (abscissa) obtained directly from the TOF measurements, are scalar quantities satisfying $p^{2} / 2=E$, where $E$ is the final-state kinetic energy. The theoretical single ionization distributions are smoothed to model the resolution of the TOF measurements. The experimental NSDI spectra have been averaged over adjacent data points $( \pm 0.12 \mathrm{au})$, yielding smoother exponential tails for comparison with theory. These are the only modifications applied to the data.

The estimates of peak laser intensities, given in Fig. 1, were deduced from experimentally determined ratios of $\mathrm{He}^{2+}$ yields to $\mathrm{He}^{+}$yields. The intensity dependence of the $390 \mathrm{~nm}$ ion yield ratios for spatially averaged $120 \mathrm{fs}$ sech pulses is known from previous theoretical work [11]. Four additional independent estimates of intensity were obtained by comparing each of the four experimental data sets of Fig. 1 with theoretical distributions calculated at a variety of intensities. Intensities associated with the best-fit theoretical spectra agree to $5 \%$ with the ion-ratio based determinations. Finally, the ratio of theoretically derived intensities $(11 / 8)$ is within $5 \%$ of those determined by experiment [12]. The calculated single ionization rate is 0.000224 au at $0.8 \mathrm{PW} / \mathrm{cm}^{2}$, and 0.000713 au at $1.1 \mathrm{PW} / \mathrm{cm}^{2}$.

At both intensities in Fig. 1 the experiment and theory are in good agreement. In particular, the calculations predict the same cutoffs observed experimentally (within experimental uncertainties) in the high momentum limit, as exponential decay sets in.

In the following we provide a more precise definition of the notion of cutoff in ionization. Toward this end, the use of constant intensity pulses in the numerics is crucial, since they result in clearly defined exponential decays at high momentum, and allow us to establish a more precise relationship between the unique intensity of the laser pulse and the corresponding cutoff momentum.

In Fig. 2 the momentum distributions are calculated using the full-dimensional two-electron integration. The pulse shapes are flat topped, ramped on and off smoothly, and varied as described above. The momentum equals $\sqrt{2} \bar{E}$, but $E$ in the double ionization case is the total kinetic energy of the two-electron wave packet, unlike Fig. 1. 


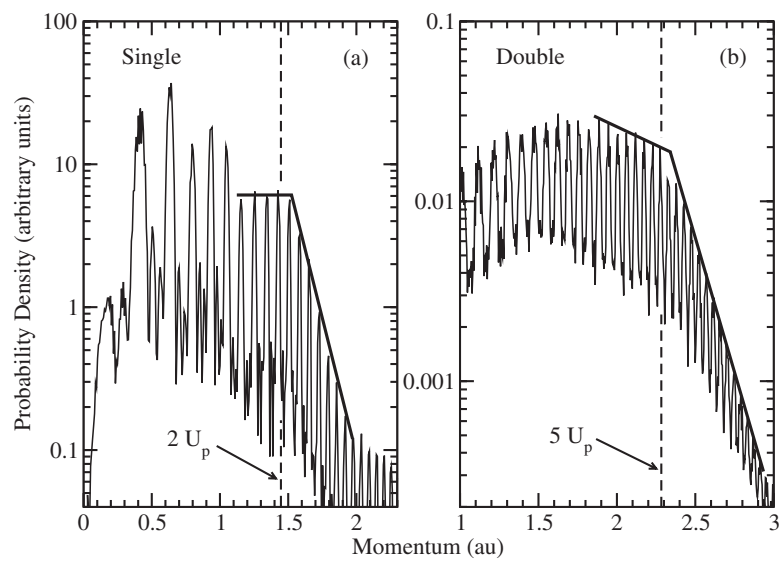

FIG. 2. Total electron momentum distributions of (a) singly and (b) doubly ionized helium. Intensity is $1.0 \mathrm{PW} / \mathrm{cm}^{2}$.

Momenta associated with kinetic energies $2 U_{p}$ and $5 U_{p}$ are indicated by dashed lines. In both spectra a plateaulike segment is followed by a region of pure exponential decay. Each region is identified by a straight line. The cutoff energy is defined as the intersection of the lines.

A cutoff energy of $2.2 U_{p}$ is evident in the single ionization distribution of Fig. 2(a). The $2 U_{p}$ threshold is well established $[12,13]$ in single ionization and corresponds to the maximum energy a "free" electron can gain from an oscillating field. The distribution beyond $2 U_{p}$ is also understood within the framework of the rescattering model. In this model, an electron released in the field can undergo approximately a $1 / 2$ cycle of propagation along a field-driven trajectory that undergoes elastic rescattering with the core. If backscattering occurs, (a low probability event), then the scattered electron can acquire as much as $10 U_{p}$ [12] from the field. The $10 U_{p}$ cutoff (not shown) is evident in both the calculated and measured spectra for single ionization.

Double ionization, Fig. 2(b), shows a similar behavior, but with a cutoff near $5.3 U_{p}$. Beyond this energy the distribution decays exponentially over 2 orders of magnitude, followed by a plateau (not shown). The plateau has an indistinct cutoff in the range $14 U_{p}$ to $20 U_{p}$.

In Fig. 3, single ionization cutoffs $(\bigcirc)$ vary little from $2.1 U p \pm 0.2 U_{p}$ as a function of intensity. The NSDI cutoffs (squares) show little variation from $5.2 U_{p} \pm 0.2 U_{p}$ until a more complicated intensity dependence develops for intensities above $I_{t 1}=1.2 \mathrm{PW} / \mathrm{cm}^{2}$. The sudden transition for $I>I_{t 1}$ suggests an abrupt change in the physical process that dominates NSDI occurs at $I_{t 1}$. Intensities $I>$ $I_{t 1}$ are not the focus of this letter since this is the realm of direct $(e, 2 e)$ rescattering, but the transition at $I_{t 1}$ is consistent with the rescattering model's basic premise that the peak energy possessed by the returning electron is $3.2 U_{p}$.

In the calculation, a signature of rescattering induced NSDI is a correlated time delay between bursts of single and double ionization. These bursts are observed by cal-

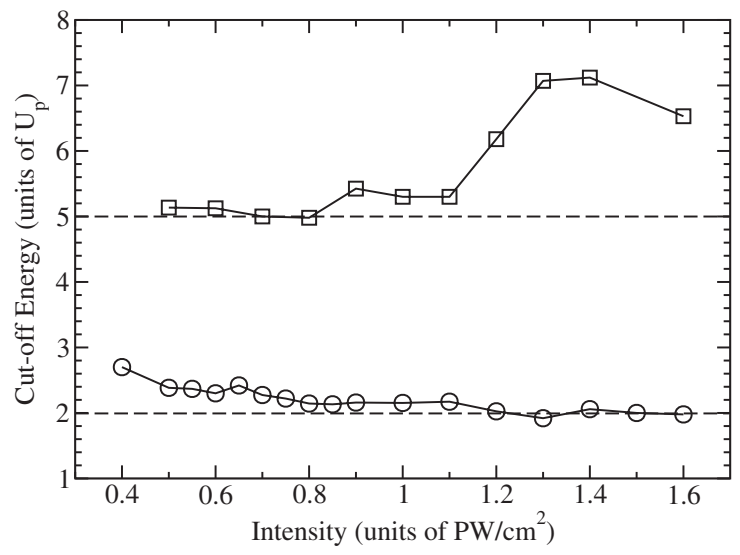

FIG. 3. Intensity dependence of single ( $\bigcirc)$ and double ( $\square$ ) ionization cutoff energies obtained from spectra described in Fig. 2.

culating the rate at which the population crosses boundaries as the singly and doubly ionizing wave packets depart the atomic core [9]. For all $I<I_{t 1}$, no double ionization is seen to occur simultaneously with single ionization, which peaks near the $E$-field maxima. The time delay observed between single and double ionization is typically about 0.8 field cycles, consistent with the rescattering model. Consequently, the time-delay calculations imply that double ionization for $I<I_{t 1}$ is a direct consequence of a rescattering event, and nonsequential.

Further insight can be obtained by examining the jointprobability distributions, like Fig. 4, over the intensity range of $0.4-1.2 \mathrm{PW} / \mathrm{cm}^{2}$. The results confirm the absence of sequential double ionization for $I<I_{t 1}$. SDI is an un-

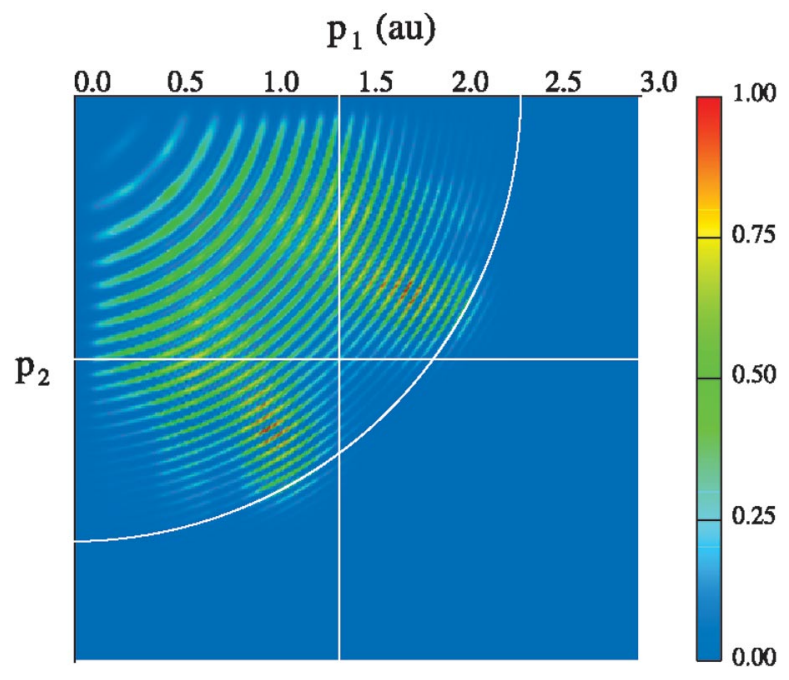

FIG. 4 (color). Joint-probability distribution in momentum space of doubly ionizing electrons at the end of a 7 field period pulse. Intensity is $I=1.0 \mathrm{PW} / \mathrm{cm}^{2}$. Along the vertical line electron 1 is constrained to kinetic energy $1.9 U_{p}: p_{1}=$ $\sqrt{3.8 U_{p}}$. Along the white circular arc total kinetic energy equals $5.3 U_{p}$. 


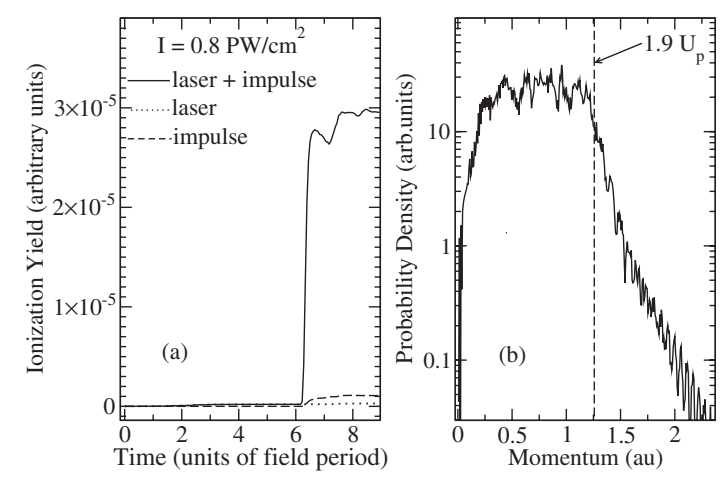

FIG. 5. (a) Response of $\mathrm{He}^{+}$to an $0.8 \mathrm{PW} / \mathrm{cm}^{2}$ laser pulse plus $E$-field impulses representing recscattering collisions. (b) The momentum distribution of the ionized electron.

correlated process that would significantly populate the space between the circular arcs of Fig. 4 [14]. Instead the arcs are due to a correlated process (double-electron above threshold ionization) in which the sum of the electrons' final-state energies is constrained to $N \hbar \omega$ above the ponderomotive shifted ground state.

In Fig. 4, a circular arc is drawn to mark the $5.3 U_{p}$ cutoff. Along this cutoff there is a prominent notchlike feature in the joint-probability distribution. One edge of the notch is marked by a vertical line at $1.9 U_{p}$ where one of the electrons (the slower electron) exhibits a cutoff that is independent of the momentum of the other. The $1.9 U_{p}$ cutoff of this slower electron is close to the $2 U_{p}$ cutoff observed in single ionization of helium ground-state, [Fig. 2(a)]. Thus a plausible interpretation of Fig. 4 is that the slower electron is the one ejected from the $\mathrm{He}^{+}(1 s)$ state. To test this identification we introduce a simple one-electron model of rescattering induced ionization of $\mathrm{He}^{+}$in which the electric field experienced by the $\mathrm{He}^{+}$bound electron during the collision is constructed explicitly. An approximation of the magnitude of the $E$ field is extracted from the full-dimensional integration by calculating $\left\langle 1 /\left|\mathbf{r}_{1}-\mathbf{r}_{2}\right|\right\rangle^{2}$ during the laser pulse. This quantity evolves in time as a series of sharply peaked impulses representing the $e-e$ collisions. The impulses are observed to peak near the zeros of the laser's electric field, consistent with the rescattering picture. When we calculate the response of the $\mathrm{He}^{+}(1 s)$ state to the sum of the laser pulse and just one of these impulses [Fig. 5(a)], we observe a burst of ionization that agrees in magnitude, duration and timing with the corresponding burst of NSDI calculated from the full two-electron integration. By contrast, neither the impulse alone, nor the laser pulse alone is capable of producing ionization yields within an order of magnitude of that observed. When the full series of impulses is applied to the $\mathrm{He}^{+}$ion, the momentum spectrum of the ionized electron has a sharp cutoff at $1.9 U_{p}$, [Fig. 5(b)], in close agreement with Fig. 4. This approach successfully models NSDI in the $I<I_{t 2}$ limit as well as for $I_{t 2}<I<I_{t 1}$.

With the slow electron identified as that ejected from the $\mathrm{He}^{+}$ion, we identify the faster as the rescattered electron. The fast electron exhibits a cutoff near $3.4 U_{p}$, (in the region along the vertical line where the slow electron retains its maximum energy of $1.9 U_{p}$ ).

Additional evidence can be extracted from the measurements by Eremina et al. [5]. Exciting argon with intense $800 \mathrm{~nm}$ pulses, they measured NSDI energy spectra at intensities below the threshold of $(e, 2 e)$ impact ionization. Applying our definition of cutoff [Fig. 2] to the $800 \mathrm{~nm} \mathrm{Ar}$ data [5], we find the argon results highly consistent with the $5.3 U_{p}$ cutoff reported here.

In summary, we have found excellent agreement between experiment and theory in a study of strong-field NSDI in the intensity regime $\left(I<I_{t 1}\right)$ where NSDI as a direct consequence of inelastic $(e, 2 e)$ rescattering is energetically forbidden. The picture that emerges from the above analysis is very different from the usual picture of NSDI $(e, 2 e)$ rescattering for $I>I_{t 1}$ in which a rescattering electron is capable of freeing the bound electron independently of the laser field by giving up much of its $3.2 U_{p}$ in an inelastic collision. We have described a new NSDI process in which one of the electrons exhibits a cutoff near $1.9 U_{p}$, independently of the other. The dynamics of this new process are captured in a simple model of $\mathrm{He}^{+}$ ionization in which the rescattering collisions are treated as electric-field impulses that are too weak to significantly ionize the $\mathrm{He}^{+}(1 s)$ independently of the laser.

Worked performed at QUB was supported by the UK EPSRC and that at The Ohio State University by NSF Contract No. PHY-0355287.

[1] P. Lambropoulos, Phys. Rev. Lett. 55, 2141 (1985).

[2] P. B. Corkum, Phys. Rev. Lett. 71, 1994 (1993).

[3] K. J. Schafer, B. Yang, L. F. DiMauro, and K. C. Kulander, Phys. Rev. Lett. 70, 1599 (1993).

[4] B. Walker et al., Phys. Rev. Lett. 73, 1227 (1994).

[5] J. Eremina et al., J. Phys. B 36, 3269 (2003).

[6] R. Lafon et al., Phys. Rev. Lett. 86, 2762 (2001).

[7] J. Chaloupka et al., Opt. Express 8, 352 (2001).

[8] V. Stert, W. Radloff, C. Schulz, and I. Hertel, Eur. Phys. J. D 5, 97 (1999).

[9] J.S. Parker, B. J.S. Doherty, K. J. Meharg, and K.T. Taylor, J. Phys. B 36, L393 (2003).

[10] J. S. Parker, E. S. Smyth, and K. T. Taylor, J. Phys. B 31, L571 (1998).

[11] J.S. Parker, L. R. Moore, D. Dundas, and K. T. Taylor, J. Phys. B 33, L691 (2000).

[12] B. Walker, B. Sheehy, K. C. Kulander, and L. F. DiMauro, Phys. Rev. Lett. 77, 5031 (1996).

[13] P. B. Corkum, N. H. Burnett, and F. Brunel, Phys. Rev. Lett. 62, 1259 (1989).

[14] J. S. Parker et al., J. Phys. B 34, L69 (2001). 